\title{
Granule Cell Suspensions Transplanted to the Lesioned Dentate Gyrus: A Model for the Growth and Reinnervation of Transplanted Neurons
}

\author{
J. Wells, D.I. Legendre, B.P. Vietje and T.S. Barett \\ Department of Anatomy and Neurobiology, University of Vermont, \\ Burlington, VT 05405, USA
}

Fluid injections into the infragranular cleavage plane of the dentate gyrus selectively lesion the granule cells. The fluid (e.g., Hanks Balanced Salt Solution) opens a cavity in the IGCP that severs the mossy fibers that cross the cavity, killing the granule cells. Few granule cells survive in the region of the lesion. If the fluid injected into the IGCP contains immature granule cells, the adult host granule cells can be killed and immediately replaced by donor granule cells. Using this model, we can follow the outgrowth of the dendrites of transplanted cells into the molecular layer (ML), quantify the compensatory changes of the dendrites as they grow into a shrinking ML and test the mechanisms of outgrowth and reinnervation.

After selective granule cell lesions or lesions plus transplants, the ML shrank precipitously during the first 2 weeks and the average thickness of the ML over the injection site was not different in transplanted animals from lesionedonly animals. By the third week, and in subsequent weeks, there was a significant increase (30\%) of the ML in transplanted compared to lesioned rats, although the ML continued to shrink. We assume that the ML in transplanted rats would shrink until it was about $25 \%$ of normal, since the number of cells surviving in a transplant was about $25 \%$ of the number in the comparable region of the normal granule cell layer. At the end of 8 weeks post-transplant, the ML was about $30 \%$ of normal, suggesting that longer post-transplant times will increase the differences between lesioned and transplanted rats.

Dendritic outgrowth was determined by injecting the transplanted cells with Lucifer Yellow (LY) in fixed sections and immunoreacting the sections with an antibody to $\mathrm{LY}$, or by immunoreacting the sections with antibodies to the dendritic protein MAP-2. At 1 week the dendrites were thin and had few spines. At 2 weeks most of the LY-filled dendrites had reached the hippocampal fissure. By 4 weeks the dendrites were thicker and had more spines per unit length than controls. At 8 weeks post-transplant the dendrites were more coiled, very thick and had about twice the number of spines $/ \mu \mathrm{m}$ than controls. The dendrites of transplanted cells were shorter than normal at each point after the transplant. The differences from controls appeared to be compensatory changes related to growing into a shrinking ML.

Some transplants were placed into the hippocampal fissure. Granule cell dendrites grew out of the transplant and into the host ML. The dendrites from the fissure transplants were inverted in the ML compared to normal granule cell dendrites. The inverted dendrites will be instructive, if they are reinnervated by the normal ML inputs. Most of the dendrites from fissure transplants grew into the ML, but, if the transplanted cells physically encroached into CA1, the dendrites could grow toward the pyramidal layer of CA1.

As an example of the usefulness of the model, we have preliminary data on one group of rats that received an antibody to NCAM along with the transplant. We analyzed the group at 4 weeks post-transplant. The average thickness of the ML was not significantly different from the lesioned-only animals. There were many LYfilled cell bodies that had no dendrites and many of the transplanted cells were tilted. The dendrites that did grow into the ML were not statistically different from the dendrites in the untreated 4-week transplants. The results suggest that there may have been a delay in the start 
of the outgrowth of the dendrites and that there may have been fewer dendrites in the ML. Additional studies at earlier post-transplant times are in progress.

In another preliminary study a group of animals received a combination of NGF and bFGF along with transplants whose cells had been labeled with bromodeoxyuridine. The transplants were analyzed after 4 weeks. These transplants were very small but still managed to rescue the ML. In this group the NGF/FGF may have prevented some of the degeneration of the host inputs to the ML, contributing to the increase in the average thickness of the ML. In both this group and the NCAM group, the potential of the model to study the mechanisms of outgrowth and reconstruction of damaged circuits is evident. 

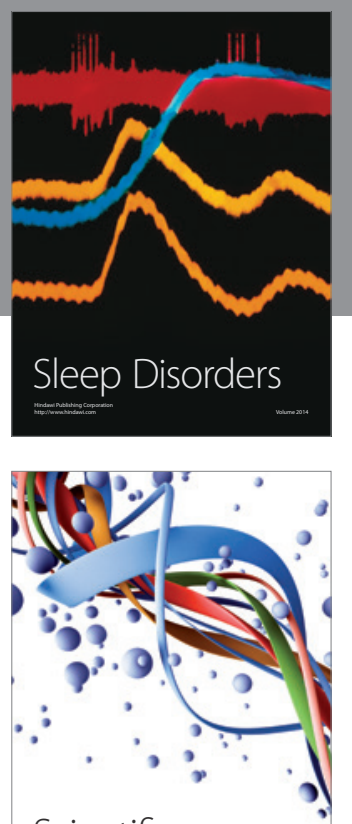

Scientifica
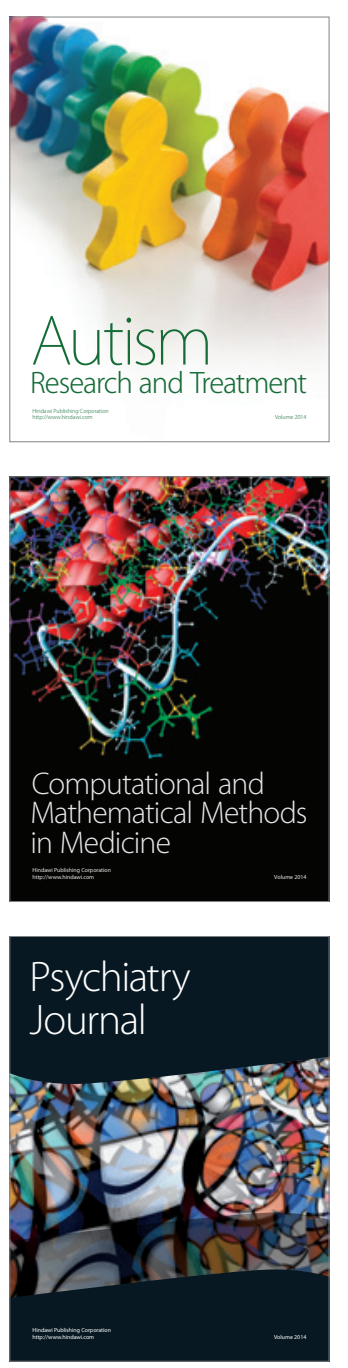
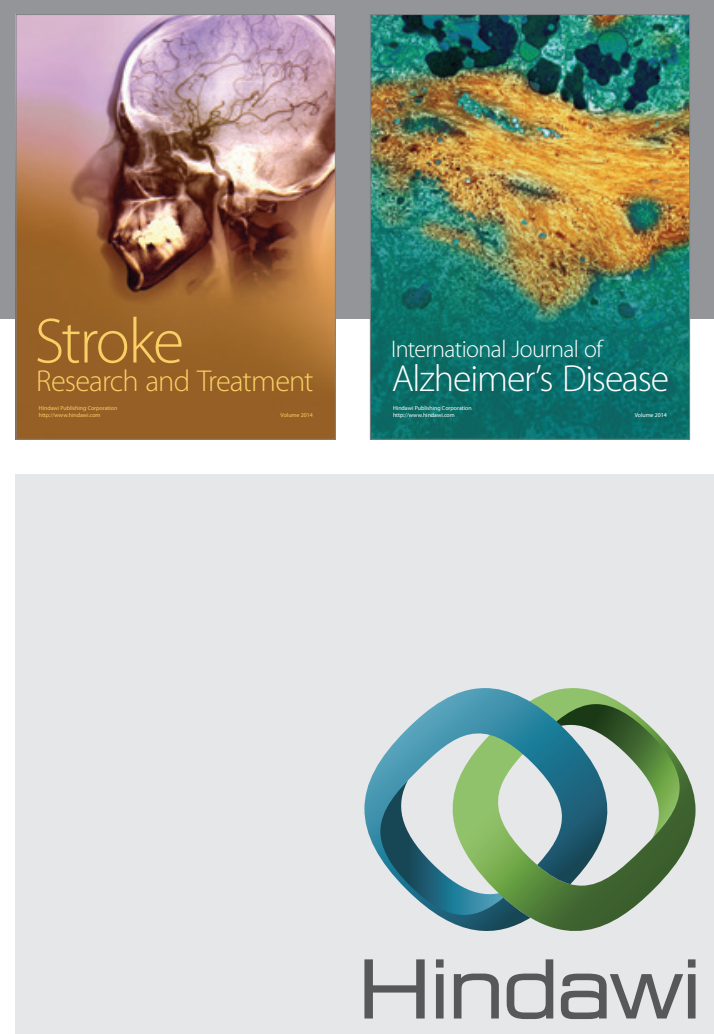

Submit your manuscripts at

http://www.hindawi.com
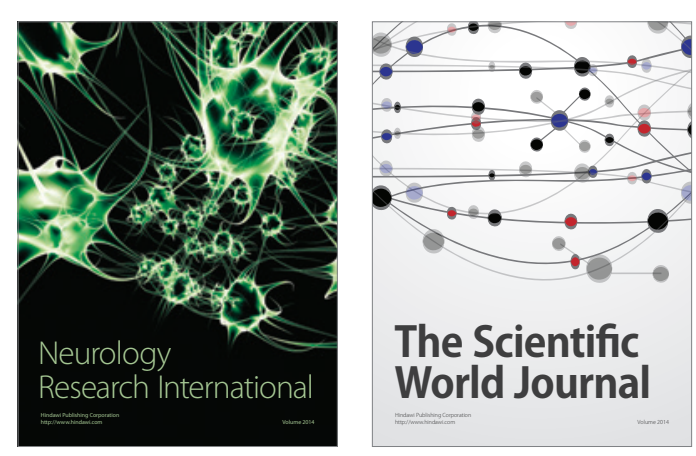

The Scientific World Journal

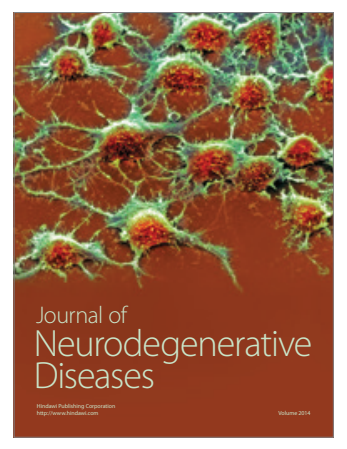

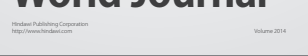

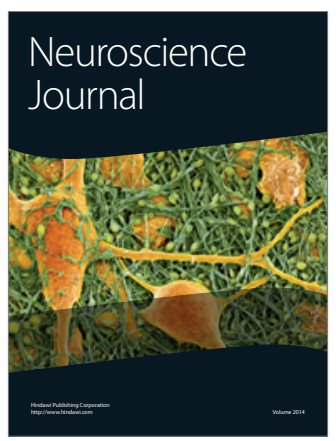

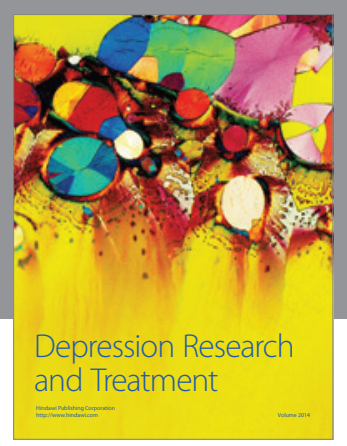
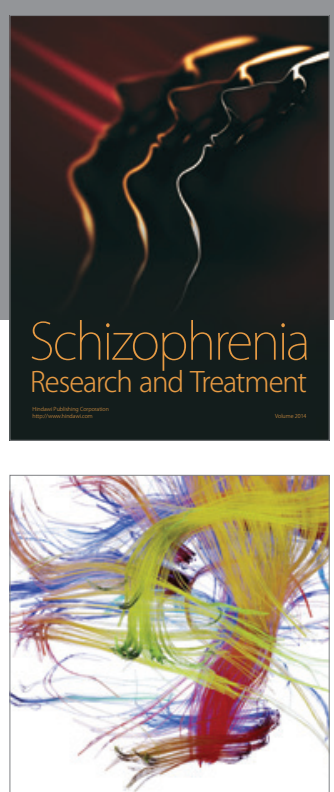

Brain Science

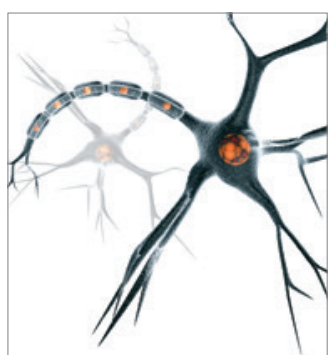

Neural Plasticity
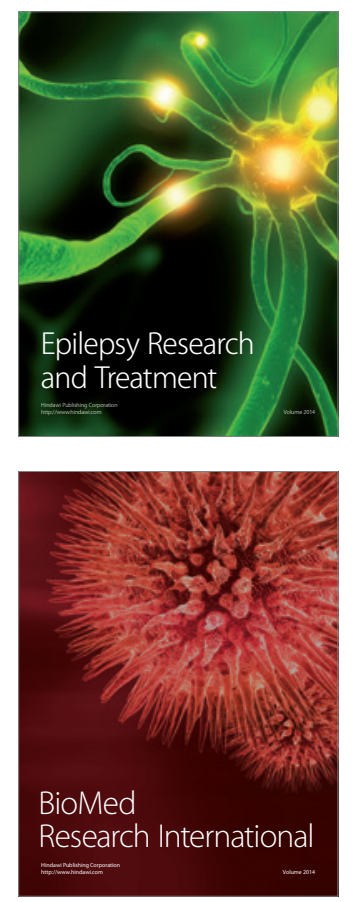

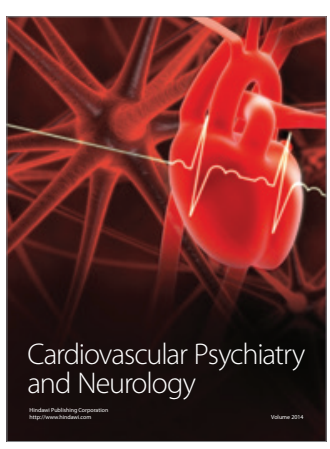

Parkinson's

Disease
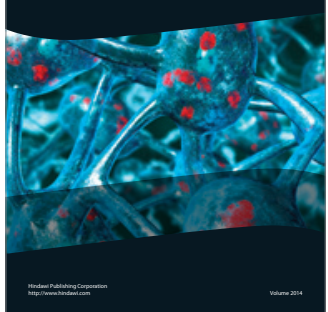